ORAL MEDICINE

\section{Oral effects of low-dose methotrexate treatment}

Kalantzis A, Marshman Z et al. Oral Surg 2005; 100: 52-62

As methotrexate is increasingly used for numerous conditions, more oral adverse effects have been recognized.

Methotrexate is a widely-used chemotherapeutic in neoplastic diseases, but also is increasingly used in low doses (LDMTX) in other circumstances, particularly rheumatoid arthritis and a variety of skin conditions. Recognised adverse effects may affect 30$80 \%$ of patients.

This paper reports 7 cases of oral ulceration ranging from nonhealing ulcers to destructive lymphoma-like lesions which occurred in patients on LDMTX. Following biopsy excision, 2 lesions in the same patient did not recur, and 5 other lesions resolved on cessation of LDMTX, which in 3 cases was restarted without recurrence in the period of follow-up. In one case a lesion had apparent clinical and histological signs of malignancy.

The authors discuss possible treatment of LDMTX-induced oral lesions, including folate supplementation which may overcome the problem without ceasing LDMTX, symptomatic treatment with topical analgesics, antiseptics or steroids, excision of lesions, and adjustment of the central treatment regime.

\section{doi:10.1038/sj.bdj.4812698}

ORTHODONTICS; DENTAL PATHOLOGY

Fluorides, orthodontics and demineralization: a systematic review

Benson PE, Shah AA et al. J Orthod 2005; 32: 102-114

Daily fluoride mouthrinse or glass ionomer cement (GIC) for bonding brackets may reduce enamel white spot lesions (WSL), but better trial evidence is needed.

WSL demineralisation is an effect of orthodontic treatment which may persist for years after treatment. This systematic review identified trials giving WSL and other assessments of demineralisation as an outcome measurement. An initial search of databases produced 191 studies which were reduced to 15 trials (10 RCTs; 5 CCTs) which met the inclusion criteria of these researchers.

Only 3 trials had both definite allocation concealment and assessor blinding, and none fulfilled all methodological assessment criteria. Risk of bias was judged low in 5 trials. On the basis of 4 trials with an estimated high risk of bias, the authors advised use of a fluoride mouthrinse until high quality trials are conducted. On the basis of 6 trials ( 3 of low bias risk), the authors considered there was some evidence that a GIC was more effective at preventing demineralization and WSL than a conventional composite resin, when used for bonding brackets.

\section{PERIODONTICS}

Post-surgical infections: prevalence associated
with various periodontal procedures
Powell CA, Mealey BL etal. JPeriodonto/ $2005 ;$ 76:329-333

Such infections are rare and there may be no benefit in prophylactic antibiotics.

This was a retrospective review of 1053 periodontal surgical procedures in 395 patients at a US military dental clinic. The techniques employed included osseous resection and grafting, flap curettage, distal wedge procedures, gingivectomy, root resections, guided tissue regeneration (GTR), implant surgery, soft tissue grafts, coronally positioned flaps, sinus augmentations and ridge preservation or augmentation. Infection was defined as increasing and progressive soft tissue swelling with presence of suppuration.

There were 8 diagnosed infections in 374 osseous resective procedures, 4 in 134 GTR procedures, 3 in 82 connective tissue grafts, 2 in 88 flap curettages, 2 in 175 implants (both stages), and 3 in other procedures. There were 8 infections in 281 procedures where antibiotics were used, and 14 in 772 where they were not. There was no statistically significant relationship of infection to any variable studied. The authors do not report the full relationship of smoking to infection in this series, although they state that 2 patients with more than 1 infection on separate occasions were not smokers.

doi:10.1038/sj.bdj.4812700

\section{ORAL DIAGNOSIS; BEHAVIOURAL SCIENCE}

\section{How do children with attention deficit} hyperactivity disorder interact in a clinical dental examination? A video analysis

Blomqvist M, Augustsson M etal. Eur J Oral Sci 2005; 113: 203-209

There is less 2-way communication in this situation.

Attention deficit hyperactivity disorder (ADHD) is currently the most commonly diagnosed developmental disorder in children. This research was part of a Swedish behavioural study in a defined population of all 555 children born in 1991 and living in a small municipality in 2001. ADHD was diagnosed in 30 boys and 5 girls. After exclusions and dropouts, 22 of these were compared with 47 controls chosen from the rest of the children.

One dentist, who was blind to the diagnosed behavioural status, examined each child in 4 stages: seating in the chair and tilting it; oral clinical examination; professional tooth cleaning and fluoride varnish application; bite-wing radiographic examination.

Regression analysis gave differences significant at the $1 \%$ level for a greater proportion of initiatives taken by ADHD children, and at the $10 \%$ level for several types of non-responsiveness in them. The authors conclude that ADHD children had less 2-way communication with the dentist, and particular difficulty in staying focused on the examination.

doi:10.1038/sj.bdj.4812701 